\title{
Predisposition to ascariasis: patterns, mechanisms and implications
}

\author{
C. V. HOLLAND* \\ Department of Zoology, School of Natural Sciences, Trinity College Dublin, Dublin 2, Ireland \\ (Received 2 Fanuary 2009; revised 26 February 2009; accepted 27 February 2009; first published online 19 May 2009)
}

\begin{abstract}
SUMMARY
Ascaris lumbricoides, the human roundworm, is a remarkably infectious and persistent parasite. It is a member of the soiltransmitted helminths or geohelminths and infects in the order of 1472 million people worldwide. Despite, its high prevalence and wide distribution it remains along with its geohelminth counterparts, a neglected disease. Ascariasis is associated with both chronic and acute morbidity, particularly in growing children, and the level of morbidity assessed as disability-adjusted life years is about 10.5 million. Like other macroparasite infections, the frequency distribution of A. lumbricoides is aggregated or overdispersed with most hosts harbouring few or no worms and a small proportion harbouring very heavy infections. Furthermore, after chemotherapeutic treatment, individuals demonstrate consistency in the pattern of re-infection with ascariasis, described as predisposition. These epidemiological phenomena have been identified, in a consistent manner, from a range of geographical locations in both children and adults. However, what has proved to be much more refractory to investigation has been the mechanisms that contribute to the observed epidemiological patterns. Parallel observations utilizing human subjects and appropriate animal model systems are essential to our understanding of the mechanisms underlying susceptibility/resistance to ascariasis. Furthermore, these patterns of Ascaris intensity and re-infection have broader implications with respect to helminth control and interactions with other important bystander infections.
\end{abstract}

Key words: Ascaris lumbricoides, epidemiology, aggregation, predisposition, susceptibility, resistance, mechanism, control.

\section{INTRODUCTION}

In his book 'Parasitism the Ecology and Evolution of Intimate Associations' Combes (2000) has eloquently described 'the inequality of host individuals in the face of parasitism'. This inequality, the epidemiological patterns that it generates and the possible mechanisms behind it, has intrigued parasitologists for many decades. More specifically, the number of macroparasites a host carries is fundamental to our understanding of helminth parasite epidemiology (Anderson, 1986). Crofton (1971), in his seminal paper on a quantitative approach to parasitism, characterized the frequency distribution of parasites in a host as clumped or over-dispersed and best described by the negative binomial. Essentially, most hosts carry few or no parasites and a small proportion of hosts carry heavy infections; these humans or other animals are sometimes described as 'wormy' (Croll and Ghadirian, 1981). The ubiquity of this distribution was demonstrated by Shaw and Dobson (1995), amongst a survey of over 270 hostmacroparasite systems, with only a single exception to the rule of aggregation.

* Corresponding author. Tel: + 353 1-8961096. E-mail: cholland@tcd.ie
This inequitable distribution has important implications for parasite transmission, strategies for control and host morbidity. Heavily infected hosts are likely to suffer greater morbidity and mortality, to make a greater contribution to the number of infective stages in the environment than hosts carrying lower intensity infections and to contribute to hostparasite regulatory processes. Consequently, developing an understanding of the mechanisms that generate susceptibility and resistance to helminth parasites, and how these generate the observed patterns of distribution is central to our understanding of parasite epidemiology and the development of appropriate strategies for control.

\section{$A S C A R I S$ AND ASCARIASIS}

In this retrospective, I have chosen to focus upon the human roundworm Ascaris lumbricoides for two reasons. Firstly, this geohelminth has been and continues to be a focus of my own research; but, perhaps more importantly, I regard it as becoming increasingly understudied relative to its counterparts Trichuris trichiura and the two hookworm species, Ancylostoma duodenale and Necator americanus.

A. lumbricoides is a remarkably infectious and persistent parasite (Crompton, 2001). An estimated 1472 million people (a quarter of the world's 
population) are infected with it and the level of morbidity, assessed as disability-adjusted life years, is about $10 \cdot 5$ million (Holland, 2005). Chronic ascariasis is known to contribute to insidious morbidity, including growth retardation and effects on cognitive development, particularly in growing children (O'Lorcain and Holland, 2000). Additionally, both A. lumbricoides and Ascaris suum, its counterpart in pigs, are known to cause liver and lung pathology as a consequence of their migration through these organs (Stephenson, 1987). Pulmonary symptoms can be pronounced and are described as Loeffler's syndrome (Loeffler, 1932, 1956). Acute ascariasis, associated with very heavy worm burdens, can result in intestinal obstruction, other serious complications and, in extreme cases, death (O'Lorcain and Holland, 2000).

Furthermore, the growing perception that infection with Ascaris and other geohelminths have a broad effect on the host's immune system, with consequences for concurrent important infectious diseases, such as malaria (Mwangi et al. 2007), renewed interest in the consequences of early infection with worms in the context of the hygiene hypothesis (Yazdanbakhsh et al. 2002) and the modulatory consequences for development of allergies (Maizels and Yazdanbakhsh, 2003), all greatly enhance the public health significance of geohelminths such as Ascaris.

In this paper, I will first provide a brief historical perspective on the phenomenon of aggregation and predisposition with respect to ascariasis. Secondly, I will evaluate the studies that have explored these epidemiological patterns and the possible mechanisms that generate them in both humans and animal model systems. Thirdly, I will outline how these patterns influenced the development of strategies for control and the broader implications of co-infections between macroparasites such as Ascaris and microparasites.

HISTORICAL PERSPECTIVE

\section{Aggregation}

The first paper to describe the frequency distribution of a number of human helminth infections was that of Croll and Ghadirian (1981). This work, based in 3 Iranian villages, described the over-dispersed distribution of Ascaris lumbricoides, Trichuris trichiura and two species of hookworm, Ancylostoma duodenale and Necator americanus, after anthelmintic treatment and worm collection. Over-dispersed frequency distributions such as these are best described by fitting the data to the negative binomial distribution and estimating the parameter $\mathrm{k}$ (Bliss and Fisher, 1953; Anderson, 1986). Values of k provide an inverse measure of the degree of over-dispersion or aggregation, with smaller values denoting greater aggregation. Anderson and May (1991) described 4 key consequences of aggregation for a parasite such as Ascaris: influence on mate-finding choices and hence egg production and transmission, densitydependent regulation of parasite populations, host morbidity and mortality in heavily infected hosts and implications for the design of effective control strategies.

\section{Predisposition}

In addition to the observations on human helminth distributions, a further important epidemiological parameter was identified during the late 1980s. Longitudinal field-based studies measured the patterns of helminth re-infection in individual patients after the provision of anthelmintic treatment and an assessment was made of the degree to which individuals re-acquired both light and heavy infection (Anderson, 1986). Worm burdens or faecal egg counts of individuals were measured and compared statistically before and after treatment, and a significant positive correlation between these was identified indicating consistency in the extent of reinfection. This phenomenon is known as predisposition. Elkins et al. (1986) were the first authors to describe predisposition to A. lumbricoides among both child and adult inhabitants of Indian villages. An important age-related pattern was also observed, with Ascaris infection declining with age as did the strength of observed predisposition.

Predisposition to ascariasis was subsequently observed in Burma (Thein-Hlaing et al. 1987), Nigeria (Holland et al. 1989), Mexico (Forrester et al. 1990), Malaysia (Chan et al. 1992) and Bangladesh (Hall et al. 1992), confirming it as a widespread phenomenon in endemic communities. In contrast, Croll and Ghadirian (1981) had failed to detect a relationship between pre- and post-treatment worm burdens in their Iranian populations. Another significant contribution to our understanding of predisposition was our demonstration, that predisposition could be maintained over multiple rounds of treatment among Nigerian children aged 5-16 years (Holland et al. 1989). In an impressive long-term study, Quinnell et al. (2001) revealed that predisposition to $N$. americanus could be detected $6-8$ years after a single round of chemotherapy, but was not detectable after repeated chemotherapy. Utilizing a different approach, Peng et al. (1998) explored natural re-infection with $A$. lumbricoides, in a rural Chinese community, over a one-year period in the absence of chemotherapy. These authors provided evidence of predisposition to light and medium intensity infections, in the absence of repeated expulsion chemotherapy.

An important review of the phenomenon of predisposition was published in 1990 and much of its content remains pertinent (and understudied) 
18 years later (Keymer and Pagal, 1990). After reviewing the existing field-based data, the authors concluded that the observed predisposition is relatively weak and factors such as sample size, method of worm intensity measurement and the duration of re-infection were important contributory factors to the sensitivity of detection. Echoing this, McCallum (1990) used probability theory to model predisposition and concluded that this epidemiological pattern is subject to the influence of both short and long-term transitory factors.

To summarize, the patterns of aggregation and predisposition for ascariasis had been well established by the late $1980 \mathrm{~s}$, and this extended to the other important geohelminths, T. trichiura (Bundy et al. 1987) and hookworm species (Schad and Anderson, 1985), in addition to multiple-species predisposition (Haswell-Elkins et al. 1987). However, the mechanisms that contribute to the observed epidemiological patterns have proved to be much more refractory to investigation. These are likely to be multi-factorial and include the relative contributions of 'exposure' versus 'susceptibility' or, as described by Combes (2000), 'the encounter filter' versus 'the compatibility filter'. Exposure can include attributes of the host, such as their behaviour, environment, socio-economic status and culture. Susceptibility can encompass host physiology (including age, sex and hormonal status), immunity and genetics, although immunity may be influenced by both genotypic and phenotypic factors such as nutrition, reproductive status or the presence of coinfections. Clearly, these relative contributions are complex, difficult to disentangle and likely to vary in both space and time.

STUDIES ON THE MECHANISMS THAT EXPLAIN AGGREGATION AND PREDISPOSITION

\section{Human subjects}

The number of studies that have attempted to unravel the factors that contribute to susceptibility and resistance to Ascaris infections in humans remains comparatively small. This is not surprising, given the logistical and particularly the ethical constraints that largely restrict human studies to correlational approaches between parameters, suspected to be involved in influencing susceptibility/resistance to infection. Furthermore, despite the pressing need for longitudinal studies of parasitology in human subjects, funding for such research remains an ongoing challenge.

In seeking explanatory mechanisms, the majority of studies have focused upon differences in susceptibility (host humoral and cellular immune response, genetics) rather than exposure, given the difficulties in measuring the later. Factors that are likely to influence the successful execution and outcome of such studies include sample size, study design, measurement of current intensity of parasite infection, length of follow-up and types of host factors investigated (Anderson, 1986; Keymer and Pagal, 1990; Holland and Boes, 2002). If a predisposition study is designed to identify individuals that demonstrate consistency in parasite intensity and assign them to a particular group, the initial sample size needs to be very large indeed in order to compensate for the relatively weak correlations that are observed between worm burdens or egg counts (Holland and Boes, 2002). This is borne out by our own experience working with Nigerian children in the late 1980s and early 1990s. Our original predisposition study commenced with 808 children at the beginning of Phase 1, fell to 580 by the third round of treatment and, after selection for consistency to predisposition status, narrowed to 120 (Holland et al. 1989, 1992; McSharry et al. 1999).

Exposure. Within the context of geohelminth infection, the measurement of exposure has proved to be consistently problematical. Wong et al. (1988) developed a method for measuring soil-derived silica in faeces as a measure of geophagia and hence a proxy for exposure to geohelminths. However, this relatively time-consuming and complex method is not appropriate for routine use. In an interesting and novel approach exploiting an invertebrate hostparasite system, Kuris et al. (2007) demonstrated how a rhizocephalan/crab host-parasite relationship could be used to explore the encounter and compatability filters. Specifically, when rhizocephalans fail to establish they leave a tell-tale record in the thoracic ganglion of the crabs, thereby providing a means of quantifying the encounter filter. Unfortunately, such tools are not easily available to us in the human geohelminth field. Henry (1988) explored the relationship between re-infection with Ascaris and risk factors for exposure in pre-school children in St Lucia. Crowding and sanitation were reported to be the most significant factors that influenced reinfection.

The humoral immune response. Several studies have focused upon the relationship between humoral immune responses and re-infection or predisposition to A. lumbricoides. The earliest study, undertaken in Venezuela, did not establish predisposition in 5 to 10-year-old children, but rather compared groups of children who did and did not exhibit re-infection (Hagel et al. 1993). A significant association between re-infection and high pre-treatment total $\mathrm{IgE}$ levels, but low parasite-specific IgE levels, was recorded. This led the authors to conclude that parasitespecific IgE may have a protective role against Ascaris infection.

In 1995, Palmer et al. undertook a case-control study among Bangladeshi children, with an average age of 9 years. A comparison of consistently 
lightly-infected versus heavily-infected subjects revealed higher concentrations of a range of antibody isotypes including parasite-specific IgG1, IgG4 and $\mathrm{IgE}$. Therefore, in contrast to the findings of Hagel and colleagues, parasite-specific IgE levels mirrored the infection intensity of the children. The authors did not rule out an effector role for these antibodies and suggested that the utilization of more specific antigens might sharpen the detection of epitopespecific protective responses.

This suggestion was borne out by our observations on the Nigerian children deemed to be predisposed to Ascaris infection. Children aged 5-16 years were identified as predisposed to remain uninfected, lightly infected and heavily infected. This selection was made by means of a statistically based cut-off and children were classified as follows: predisposed to remain uninfected (no worms on either of the two occasions), those with consistently light infections (1-24 worms in Phase 1 and 1-8 worms in Phase 2), and those with consistently heavy infections $(>25$ worms at Phase 1 and $>9$ worms at Phase 2) (Holland et al. 1989). The 3 groups of children were compared using a range of serum factors and 3 different sources of Ascaris antigen were utilized.

Only the defined allergen, Ascaris ABA-1 as a bacterial recombinant protein, provided evidence for a significant relationship between predisposition status and parasite-specific IgE. The putatively immune group tended to have higher levels of rABA-1 specific IgE, compared to the susceptible group that had low levels. In addition, higher levels of inflammatory indicators were detected in the putatively immune group (serum ferritin, eosinophil cationic protein and C-reactive protein) (McSharry et al. 1999). This finding is of particular interest with respect to our recent findings in the mouse model (see below).

Cytokine responses. The first data on cytokine responses in humans infected with Ascaris were obtained by Cooper and colleagues (2000), who described a sample of young Ecuadorian adults with moderate Ascaris infections compared to uninfected control subjects. Infected individuals manifested a highly polarized Th2 response with a predominance of IL-4 and IL-5. Two important recent studies have followed concerning the influence of cytokines upon infection intensity and re-infection in Cameroonian children and adults (Turner et al. 2003; Jackson et al. 2004). In contrast to data from laboratory models (Urban et al. 1991; Richard et al. 2000; Schopf et al. 2002), no evidence for a role for Th2 cytokines in protective immunity in humans had been previously provided.

In the first study, the relationship between cytokine production and intensity of $A$. lumbricoides was investigated in Cameroonian children and adults aged 2-36 years (Turner et al. 2003). Intensity was measured by means of egg counts and subjects were divided into child and adult age classes. Evidence was provided that Th2 cytokines, and in particular IL-9, IL-10 and IL-13, are associated with reduced parasite intensity in older children and adults. The authors concluded that their data were consistent with the hypothesis that Th2 cytokines have a role in mediating Ascaris intensity. Evidence from murine models suggests that this control may be via changes in intestinal environment that may impinge upon both larval and adult survival (Turner et al. 2003).

In a second study, the egg counts of $A$. lumbricoides (and $T$. trichiura) were recorded before and after treatment amongst children and adults aged 4-57 years (Jackson et al. 2004). Egg counts for A. lumbricoides were recorded pre- $(n=191)$ and posttreatment $(n=135), 8-9$ months later. Because of the level of complexity of the data, a principal components analysis was required to explore the multivariate variation in both parasitological and cytokine data sets. The results from this paper added weight to their earlier observations and confirmed the importance of Th2 cytokines in immunity to ascariasis.

After controlling for age and gender, Th2 cytokines were significantly negatively associated with re-infection and this association was particularly pronounced for IL-5 and IL-13. The authors believe that their observations suggest that persistent susceptibility is, at least in part, due to variation in Th2driven responses. In contrast to the earlier paper by Turner, the effects were more pronounced in younger children. This led the authors to suggest that heterogeneity in cytokine responses may operate differently depending upon the geographical location of the study. This may be due to differences in transmission patterns or even historical differences in parasite dynamics. The authors conclude that these age and location-related differences may have implications for the differential impact of de-worming programmes on immune responses.

Genetic studies of susceptibility to ascariasis. Williams-Blangero and colleagues have been at the forefront of the investigation of the genetic epidemiology of infectious disease in general and ascariasis in particular. Their studies of the Jirel population in Nepal represent a unique context within which the conduct of detailed pedigree studies provides an opportunity to disentangle environmental from genetic effects on the phenotype of Ascaris intensity.

Prior to the Jirel investigations, relatively little work had been undertaken on the relationship between susceptibility to ascariasis and host genetics. Familial predisposition had been established by Forrester et al. (1990) in Mexico and Chan and colleagues in Malaysia (1994a). However when Chan et al. (1994b) explored the correlation between parasite intensities within families they failed to detect a significant role for host genetic factors. We 
undertook an associational study on the distribution of the major histocompatability complex (MHC) alleles in HLA-A, HLA-B and HLA-C among the same groups of Nigerian children that had been selected for their predisposition status (Holland et al. 1992). None of the children who were predisposed to remain uninfected possessed the HLA-A30/31 alleles, and the frequency of occurrence of this haplotype was significantly higher in the children observed to be consistently re-infected.

Arguing that association studies of candidate genes can lead to false-positive results, Williams-Blangero et al. (1999) reported the results of their first study of 1261 Jiral individuals belonging to a single pedigree. Individuals were assessed for Ascaris intensity (both egg counts and worm burden) at 2 time-points, posttreatment follow-up occurred 1 year after treatment. This analysis demonstrated that between 30 and 50\% of the variation in worm burden of Ascaris was due to genetic factors.

These authors have recently published a wholegenome scan of 1258 members of the Jirel population (Williams-Blangero et al. 2008). This represents a refinement of an earlier study of 444 individuals (Williams-Blangero et al. 2002), and, provides greater statistical power. These 2 later studies, measured susceptibility to Ascaris infection by means of faecal egg counts at the same time-point (i.e. not with respect to re-infection as in their first study). Prevalence values for Ascaris between the 3 studies range from $21 \cdot 3 \%$ to $27 \cdot 2 \%$. A variance component linkage analysis was used to measure the covariance between infection and genotype, using microsatellite markers at approximately $10 \mathrm{cM}$ intervals among individuals in a family, and hence to identify quantitative trait loci (QTL). The genome scan revealed 6 chromosomal regions with evidence of QTLs influencing intensity of infection. Three significant QTLs and 3 suggestive QTLs were identified on chromosomes $13,11,8$ and 1 respectively. Future work is likely to focus upon the identification of specific candidate genes underlying the 3 significant QTLs, 2 of which were identified in the previous scan $(13 q$ and $8 \mathrm{q}$ ). The peak on chromosome 13 is close to the known locus of a major candidate gene, TNFSF13B, involved in the regulation of $\mathrm{B}$ cell activation and immunoglobulin secretion (Yan et al. 2000).

\section{Animal models}

Keymer and Pagal had stated in 1990 that sufficient data on predisposition were available for the erection and testing of specific hypotheses concerning causal mechanisms. Given the logistical and ethical constraints operating on human studies, they advocated the use of laboratory models, where variables such as nutritional status, genetic background, immunocompetence and behaviour can be manipulated experimentally.
Given the difficulties in the conduct of human studies, outlined above, animal models of aggregation and predisposition clearly have many obvious advantages in terms of the degree of control that we can employ. The availability of defined inbred rodents (e.g. congenic and recombinant, knockout and transgenic strains) and an array of immunological reagents, make rodent models of disease an attractive option for the investigator seeking to unravel cause and effect. However, with respect to ascariasis, we lack a natural rodent model system in which the parasite completes its life cycle in a manner similar to that of humans. This is in marked contrast to Trichuris muris, the murine species is closely related and has a similar host-parasite relationship to that of its human counterpart, T. trichiura. The dissection of the immune responses to T. muris, in inbred and knockout mice, has been highly informative (Cliffe and Grencis, 2004). Heligimosomoides bakeri has proved to be an excellent model organism for nematodes of importance in livestock (see Behnke et al. 2003 ; and current issue Behnke et al. 2009). An enduring example of the use of an animal model to explore the generative mechanisms of aggregation and predisposition was employed by Tanguay and Scott (1992) utilizing both inbred and outbred mice infected with $H$. bakeri. The investigators were able to simultaneously explore host behaviour, genetics and acquired resistance using a controlled exposure regime.

The mouse model. However, comparative studies on larval migration in pigs and mice have led to a revised view of the mouse as a suitable model for exploring susceptibility to the early phase of Ascaris infection (Murrell et al. 1997; Slotved et al. 1998). As discussed previously, we do not fully understand the mechanisms of predisposition, but it is likely that events early in infection determine the subsequent status of the host in terms of susceptibility/resistance. It has been suggested that pulmonary migration by Ascaris larvae could create a highly polarized Th2 immune environment in the lung mucosa, and genetic variation in the host response at this level may influence the course of infection (Cooper et al. 2000). Early work by Mitchell et al. (1976) had demonstrated variability in the susceptibility of different strains of inbred mice to ascariasis.

After optimization of the methodologies for infection and recovery, we have identified a mouse model for susceptibility and resistance to early Ascaris infection (Lewis et al. 2006). C57BL/6j mice are consistently highly susceptible to Ascaris larvae in the lungs, peaking at day 7 post-infection, in contrast to $\mathrm{CBA} / \mathrm{Ca}$ mice that remain consistently resistant to lung infection. Subsequently, we used this model to assess the significance of inflammatory processes within the murine lung (leucocyte population in bronchalveolar lavage (BAL) fluid and lung 
histopathology) (Lewis et al. 2007). We concluded that the responses mirrored larval intensity and that the pulmonary inflammatory immune response was not prominently involved in primary protection of mice to Ascaris infection in the latter days of infection in the lungs.

The lack of support for a pulmonary mechanism led us to suggest that a hepatic/post-hepatic factor, that varies between $\mathrm{C} 57 \mathrm{BL} / 6 \mathrm{j}$ and $\mathrm{CBA} / \mathrm{Ca}$ mice, may play a critical role in the successful migration through host tissues. Our most recent data, concerning the comparative histo-pathological hepatic inflammatory response in $\mathrm{C} 57 \mathrm{BL} / 6 \mathrm{j}$ and $\mathrm{CBA} / \mathrm{Ca}$ mice, have revealed an important difference in the timing of the response between the two strains (Dold et al. 2008). Specifically, in resistant CBA mice the most pronounced response occurred on day 4 of infection, this coincides with the migration of lower larval numbers from the liver to the lungs. In contrast, the severe inflammatory response in $\mathrm{C} 57 \mathrm{BL} / 6 \mathrm{j}$ mice took place on day 6 post-infection, a time by which most larvae have already, successfully migrated to the lungs. Therefore, these data suggest a possible inflammatory mechanism of resistance to larval Ascaris migration, acting rapidly and focused in the liver.

To conclude, we are now confident that we have a model of differential hepatic inflammation that we can use to tease out the potential mechanisms that influence susceptibility and resistance to larval ascariasis. This has both public health and veterinary significance, given that we know that liver inflammation occurs in both humans (Javid et al. 1999) and pigs (Perez et al. 2001; Frontera et al. 2003). Furthermore, for ethical reasons we know virtually nothing about the dynamics and impact of larval migration in humans; and in pigs, we still lack the versatility provided by inbred and knockout mice.

The pig model. Nevertheless, aggregation and predisposition have been modelled successfully in outbred pigs and epidemiological patterns in pigs have been described that were analogous to those of A. lumbricoides in humans (Boes et al. 1998). Furthermore, as argued by Coates (2000) the sample sizes utilized to establish predisposition were much smaller than those required for human studies. Roepstorff et al. (1997) infected pigs with 3 doses of Ascaris eggs and were able to demonstrate that although each pig became infected, an expulsion event occurred between day 14 and day 21 post-infection that resulted in an overdispersed distribution of adult Ascaris worms.

These observations indicate the considerable potential of the Ascaris-pig model to explore the mechanistic basis of the observed aggregation and predisposition particularly as exposure can be controlled or explored using natural and trickle infection protocols. Furthermore, in exciting and novel work,
Nejsum et al. (2009a) have recently reported a study that explored the dynamics of genetically marked $A$. suum infections in pigs. The authors described significant differences in the distribution, abundance and size of a variety of worm genotypes among individual pigs. This confirms the idea that genetic heterogeneity among parasites as well as among hosts, plays an important role in determining the success of infection. Other work by Nejsum and colleagues $(2009 b)$ on host genetics, confirmed that heritability estimates in the Ascaris-pig model were similar to those estimated in humans by Williams-Blangero (1999, 2002, 2008). These observations demonstrate the potential of identifying the underlying QTL using the Ascaris-pig model system.

THE SIGNIFICANCE OF GENETIC VARIATION IN HELMINTH PARASITES

Genetic variation in helminth parasites has received comparatively less attention than heterogeneity in host responses. Such polymorphism is likely to have profound epidemiological effects and is essential for host-parasite co-evolution (Read and Viney, 1996). Furthermore, there are important public health implications including the influence of parasite variation upon the development of anti-helminth vaccines and anthelmintic resistance (Maizels and Kurniawan-Atmadja, 2002). Certainly, helminth genetics are likely to be an important contributor to host responsiveness and this has been demonstrated for some helminth species including T. muris (Bellaby et al. 1996).

Considerable attention has been focused upon the investigation of human and pig Ascaris using genetic markers to explore cross-infection and hybridization and microspatial genetic variation within Ascaris populations that may shed light upon modes of transmission (reviewed by Anderson et al. 1998). Anderson et al. (1995) identified considerable genetic diversity within populations of worms infecting humans-41 distinct mitochondrial genotypes in over 200 worms. Furthermore, certain parasite genotypes were found more frequently than expected by chance in certain hosts. However, there is a paucity of data on genetic variation in Ascaris and its relationship with host responsiveness.

Fraser and Kennedy (1991) examined heterogeneity in the expression of surface antigens of A.lumbricoides infective larvae by assessing variation in antibody binding taken from the same population from whom the infective eggs were collected. Considerable heterogeneity was observed between larvae in their capacity to bind to antibody from a given donor. The authors suggested that this might reflect quantitative differences arising from surface antigen polymorphism or qualitative effects from differential gene expression. It has been suggested that the 
marked differences in the fecundity of $A$. lumbricoides observed between Nigeria and Bangladesh (Hall and Holland, 2000) may reflect parasite differences in parasite immunogenicity (Wakelin and Bradley, 2002). What is certain is that the contribution of parasite genetics remains an understudied component of the mechanisms that explain host susceptibility and resistance to ascariasis and other helminth infections

IMPLICATIONS OF THESE EPIDEMIOLOGICAL PATTERNS FOR PARASITE CONTROL

The practical implications of the observed frequency distribution of, and predisposition to, human helminth infections are significant and not only for host morbidity but also for the development of the most effective strategies for control (Anderson and Medley, 1985; Anderson and May, 1991). Specifically, the hypothesis to be tested was whether the treatment of heavily infected individuals within a community would not only reduce morbidity and mortality in the treated group, but also modify the transmission dynamics of the community as a whole. This so-called 'selective approach' was viewed as a potentially attractive option because the use of relatively few doses of chemotherapeutic drugs, might reduce costs and the likelihood of the development of anthelmintic resistance (Crompton, 1994).

We compared universal, targeted and selective treatment for ascariasis in Nigerian villages using levamisole (Asaolu et al. 1991) and subsequently evaluated the cost-effectiveness of these approaches (Holland et al. 1996). The universal approach involved the treatment of all willing members of the community with the exception of pregnant women and children under the age of 2 years. The targeted approach consisted of children aged 5-15 years receiving treatment and the selective approach provided chemotherapy to $20 \%$ of the population with the heaviest Ascaris infections (Asaolu et al. 1991). Targeted treatment of school-age children proved to be an attractive option, in terms of impact of treatment upon a high-risk group, reduction in intensity among untreated adults and cost-effectiveness in comparison to the universal approach. Furthermore, the selective option proved to be difficult to administer, costly and unpopular with the community.

Hall and colleagues (1992) observed that the disadvantages of the selective approach included the necessity to identify heavily infected people before they are treated and the likelihood of re-infection, particularly heavy infection, among such subjects was likely to occur over a relatively short period. Other studies also reported reductions in the prevalence and intensity of ascariasis in untreated adults within communities where children had been targeted for treatment (Thein Hlaing et al. 1991; Bundy et al. 1990).
Subsequently, the World Health Organization have placed the treatment of school-age children at the heart of its commitment to the control of soiltransmitted helminths, with a target of regular treatment of at least $75 \%$ of school-age children by 2010 (Albonico et al. 2006). Helminth control within schools has proved to be highly effective in developing countries, with trained teachers distributing the drugs in combination with the provision of health education (Montresor et al. 2002).

More recently, the epidemiology and impact of soil-transmitted helminths in pre-school children has come into focus and a WHO informal consultation (2002) recommended that pre-school children over 1 year old should be included in regular deworming programmes. Furthermore, the consultation and other commentators (Mwangi et al. 2007; Albonico et al. 2008), have specifically highlighted the need to define the interactions between geohelminths and important co-infections, such as malaria, in these young children, particularly if they are to be included in large-scale de-worming programmes. We have recently undertaken a randomized placebo-controlled intervention study on the impact of 4-monthly anthelmintic treatment (with albendazole) on ascariasis in Nigerian children aged 1-5 years as part of a wider study to assess the impact of anthelmintic treatment upon the prevalence and acquisition of malaria. A. lumbricoides proved to be the most prevalent geohelminth among this age group (48\%) and repeated 4-monthly treatment with albendazole was effective in reducing prevalence and intensity of infection (Kirwan et al. 2009). Clearly we require good quality data (from a range of geographical locations) on the epidemiology and impact of helminth infections in these younger children and appropriate strategies for their inclusion in control programmes analogous to what we have obtained for school-age children (WHO, 2002; Albonico et al. 2008).

\section{CONCLUDING REMARKS}

Ascariasis, despite its global distribution and the sheer numbers of individuals it infects, remains a classic neglected disease (Hotez et al. 2007). Furthermore, it is not an infection that exists alone but is found in the company of other geohelminths, particularly Trichuris (Holland et al. 1989; Tchuente et al. 2003) and important microparasitic diseases such as malaria and HIV. This polyparasitism leads to important interactions both in terms of pathogenesis and immunity. The field of Ascaris epidemiology and, specifically, that of understanding the mechanisms responsible for the observed aggregation and predisposition, is undoubtedly a challenging one. On the one hand, we are faced with the difficulties inherent in working with human subjects who live in challenging environments and asking them 
to participate in longitudinal studies with potentially multiple interventions and measurements. On the other hand, we look to the more refined animal model systems for greater control and ease of manipulation, but these vary in what we can derive from them.

Undoubtedly, significant progress has been made and parallel observations in humans and other animals have provided us with important lines of enquiry to pursue. This is exemplified by the correspondence between our observations of elevated inflammatory indicators in putatively immune Nigerian subjects and the differential hepatic inflammation in a susceptible and resistant strain of inbred mouse. This emphasizes how selected phases of infection, specifically the role of the liver migratory phase, are tractable within a murine model for ascariasis. In addition, observations on cytokine responses in Cameroonian subjects infected with Ascaris support observations made in other murine models of helminth susceptibility. This underlines the importance of undertaking both field-based studies in human subjects (involving both epidemiologists and immunologists) in tandem with laboratory-based animal model investigations.

Furthermore, the demonstration of genetic control of the intensity of ascariasis raises a number of questions. Work in humans has now established the QTL for intensity of infection within a unique human population and the identification of specific genes is likely to follow. However, it is of interest to speculate as to how widespread this heritability is likely to be in different geographical locations and under different conditions of transmission and regulation. Quinnell (2003) suggested that heritability of resistance to hookworms might be higher in environments where exposure is relatively homogenous. Clearly, studies of what Petri et al. (2008) refer to as human genetic epidemiology, in the context of infectious disease, require investigation in a variety of environments that vary in helminth transmission and epidemiology. The development of the pig-Ascaris model now provides a superb opportunity to explore the interaction between host and parasite genetics utilizing a high degree of experimental manipulation and control.

The observation by Turner and colleagues (2003) whereby apparent plasticity of age-specific cytokine responses is observed between different populations reveals another layer of complexity that requires excavation. This suggests the need to be aware of the impact of de-worming upon the immune responses of different human populations living under different regimes of helminth intensity and regulation. Furthermore, exploration of the interplay between helminth infections, immune responses to their presence and that of other important bystander infections, and their control by chemotherapy, remains one of the most crucial challenges facing parasite epidemiologists and immunologists.
I am most grateful to Jerzy Behnke, Chris Dold, Patrick Kirwan, Malcolm Kennedy and Rory O'Donnell for their helpful comments on early drafts of this paper.

This paper is dedicated to Lani Stephenson, a pioneer in field-based helminth studies.

\section{REFERENCES}

Albonico, M., Allen, H., Chitsulo, L., Engels, D., Gabrielli, A.-F. and Savioli, L. (2008). Controlling soil-transmitted helminthiasis in pre-school age children through preventive chemotherapy. PLOS Neglected Tropical Diseases 2, e126.

Albonico, M., Montresor, A., Crompton, D. W. T. and Savioli, L. (2006). Intervention for the control of soil-transmitted helminthiasis in the community. Advances in Parasitology 61, 311-348.

Anderson, R. M. (1986). The population dynamics and epidemiology of intestinal nematode infections. Transactions of the Royal Socity of Tropical Medicine and Hygiene 80, 686-696.

Anderson, R. M. and May, R. M. (1991). Infectious Diseases of Humans. Dynamics and Control. Oxford University Press, Oxford, UK.

Anderson, R. M. and Medley, G. (1985). Community control of helminth infections of man by mass and selective chemotherapy. Parasitology 90, 629-660.

Anderson, T. J. C., Blouin, M. S. and Beech, R. N. (1998). Population biology of parasitic nematodes: applications of genetic markers. Advances in Parasitology 41, 219-283.

Anderson, T. J. C., Romero-Abal, M. E. and Jaenike, J. (1995). Mitochondrial DNA and Ascaris microepidemiology: the composition of parasite populations from individual hosts, families and villages. Parasitology 110, 221-229.

Asaolu, S. O., Holland, C. V. and Crompton, D. W. T. (1991). Community control of Ascaris lumbricoides in rural Oyo State, Nigeria: mass, targeted and selective treatment with levamisole. Parasitology 103, 291-298.

Behnke, J. M., Iraq, F., Menge, D., Baker, R. L., Gibson, J. and Wakelin, D. (2003). Chasing the genes that control resistance to gastrointestinal nematodes. Fournal of Helminthology 77, 99-109.

Bellaby, T., Robinson, K. and Wakelin, D. (1995). Induction of differential T-helper-cell responses in mice infected with variants of the parasitic nematode Trichuris muris. Infection and Immunity 64, 791-795.

Bliss, C. I. and Fisher, R. A. (1953). Fitting the negative binomial distribution to biological data. Biometrics 9, 176-199.

Boes, J., Medley, G. F., Eriksen, L., Roepstorff, A. and Nansen, P. (1998). Distribution of Ascaris suum in experimentally and naturally infected pigs in comparison with Ascaris lumbricoides infections in humans. Parasitology 177, 589-596.

Bundy, D. A. P., Cooper, E. S., Thompson, D. E., Didier, J. M., Anderson, R. M. and Simmons, L. (1987). Predisposition to Trichuris trichiura in humans. Epidemiology and Infection 98, 65-71.

Bundy, D. A. P., Wong, M. S., Lewis, L. L. and Horton, J. (1990). Control of geohelminths by delivery of targeted chemotherapy through schools. Transactions of the Royal Society of Tropical and Medicine and Hygiene 84, 115-120. 
Chan, L., Bundy, D. A. P. and Kan, S. P. (1994a). Aggregation and predisposition at the familial level. Transactions of the Royal Society of Tropical and Medicine and Hygiene 88, 46-48.

Chan, L., Bundy, D. A. P. and Kan, S. P. (1994b). Genetic relatedness as a determinant of predisposition to Ascaris lumbricoides and Trichuris trichiura infection. Parasitology 108, 77-80.

Chan, L., Kan, S. P. and Bundy, D. A. P. (1992). The effect of repeated chemotherapy on age-related predisposition to Ascaris lumbricoides and Trichuris trichiura. Parasitology 104, 371-377.

Cliffe, L. J. and Grencis, R. K. (2004). The Trichuris muris system: a paradigm of resistance and susceptibility to intestinal nematode infection. Advances in Parasitology 57, 255-307.

Coates, S. (2000). Modelling the population dynamics of Ascaris suum in pigs. Ph.D. thesis. University of Warwick, Coventry, UK.

Combes, C. (2001). Parasitism the Ecology and Evolution of Intimate Interactions. The University of Chicago Press, Chicago, IL, USA.

Cooper, P. J., Chico, M. E., Sandoval, C., Espinel, I., Guevara, A., Kennedy, M. W., Urban, J. F. Jr., Griffin, G. E. and Nutman, T. B. (2000). Human infection with Ascaris lumbricoides is associated with a polarized cytokine response. The Fournal of Infectious Diseases 182, 1207-1213.

Crofton, H. D. (1971). A quantitative approach to parasitism. Parasitology 63, 343-364.

Croll, N. A. and Ghadirian, F. (1981). Wormy persons: contributions to the nature and patterns of overdispersion with Ascaris lumbricoides, Ancylostoma duodenale, Necator americanus and Trichuris trichiura. Tropical Geographical Medicine 33, 241-248.

Crompton, D. W. T. (1994). Ascaris lumbricoides. In Parasitic and Infectious Diseases Epidemiology and Ecology (ed. Scott, M. E. and Smith, G.), pp. 175-196. Academic Press, San Diego, CA, USA.

Crompton, D. W. T. (2001). Ascaris and ascariasis. Advances in Parasitology 48, 285-375.

Dold, C., Cassidy, J. P., Behnke, J. M. and Holland, C. V. (2008). Exploiting the Ascaris suum mouse model to identify the mechanistic basis of resistance/ susceptibility. Abstracts of the Proceedings of the Xth European Multicolloquim of Parasitology, Paris August 24-28, p. 50.

Elkins, D., Haswell-Elkins, M. R. and Anderson, R. M. (1986). The epidemiology and control of intestinal helminths in the Pulicat region of Southern India. I Study design and pre- and post-treatment observations on Ascaris lumbricoides infection. Transactions of the Royal Society of Tropical Medicine and Hygiene $\mathbf{8 0}$, 774-792.

Fraser, E. M. and Kennedy, M. W. (1991). Heterogeneity in the expression of surface-exposed epitopes among larvae of Ascaris lumbricoides. Parasite Immunology 13, 219-225.

Forrester, J. E., Scott, M. E., Bundy, D. A. P. and Golden, M. H. N. (1990). Predisposition of individuals and families in Mexico to heavy infection with Ascaris lumbricoides and Trichuris trichiura. Transactions of the Royal Society of Tropical Medicine and Hygiene 84, 272-276.
Frontera, E., Roepstorff, A., Gazquez, A., Reina, D., Serrano, F. J. and Navarrete, I. (2003).

Immunohistochemical distribution of antigens in liver of infected and immunized pigs with Ascaris suum.

Veterinary Parasitology 111, 9-18.

Hagel, I., Lynch, N. R., Di Prisco, M. C., Rojas, E., Perez, M. and Alvarez, N. (1993). Ascaris reinfection of slum children: relation with the $\mathrm{IgE}$ response. Clinical and Experimental Immunology 94, 80-83.

Hall, A., Anwar, K. S. and Tomkins, A. (1992). Intensity of reinfection with Ascaris lumbricoides and its implications for parasite control. Lancet 339, 1253-1257.

Hall, A. and Holland, C. V. (2000). Geographical variation in Ascaris lumbricoides fecundity and its implications for helminth control. Parasitology Today 61, 540-544.

Haswell-Elkins, M. R., Elkins, D. B. and Anderson, R. M. (1987). Evidence for predisposition in humans to infection with Ascaris, hookworm, Enterobius and Trichuris in a South Indian fishing village. Parasitology 95, 323-337.

Henry, F. J. (1988). Reinfection with Ascaris lumbricoides after chemotherapy: a comparative study in three villages with varying sanitation. Transactions of the Royal Society of Tropical Medicine and Hygiene 82, 460-464.

Holland, C. V. (2005). Ascaris, Trichuris, hookworm and Enterobius. In Topley and Wilson's Parasitology, 10th edn, pp. 712-736. Hodder Arnold, UK.

Holland, C. V., Asaolu, S. O., Crompton, D. W. T., Crichton, W. B., Torimiro, S. E. A. and Walters, D. E. (1992). A possible genetic factor influencing protection from infection with Ascaris lumbricoides. Fournal of Parasitology 78, 915-916.

Holland, C. V., Asaolu, S. O., Crompton, D. W. T., Stoddart, R. C., MacDonald, R. and Torimiro, S. E. A. (1989). The epidemiology of Ascaris lumbricoides and other soil-transmitted helminths in primary school children from Ile-Ife, Nigeria. Parasitology 99, 275-285.

Holland, C. V. and Boes, J. (2002). Distributions and predisposition: people and pigs. In The Geohelminths : Ascaris, Trichuris and Hookworm (ed. Holland, C. V. and Kennedy, M. W.), pp. 1-24. Kluwer Academic Publishers, The Netherlands.

Holland, C., O'Shea, E., Asaolu, S. O., Turley, O. and Crompton, D. W. T. (1996). A cost-effective analysis of anthelmintic intervention for community control of soil-transmitted helminth infection: Levamisole and Ascaris lumbricoides. Fournal of Parasitology 82, 527-530.

Hotez, P. J., Molyneux, D. H., Fenwick, A., Kumaresan, J., Ehrlich Sachs, S., Sachs, J. and Savioli, L. (2007). Control of neglected tropical diseases. New England Fournal of Medicine 357, 1018-1027.

Jackson, J. A., Turner, J. D., Rentoul, L., Faulkner, H., Behnke, J. M., Hoyle, M., Grencis, R. K., Else, K. J., Kamgno, J., Boussinesq, M. and Bradley, J. E. (2004). T helper cell type 2 responsiveness predicts future susceptibility to gastrointestinal nematodes in humans. The Fournal of Infectious Diseases 190, 1804-1811.

Javid, G., Wani, N. A., Gulzar, G. M., Khan, B. A., Shah, A. H., Shah, O. J. and Khan, M. (1999). 
Ascaris-induced liver abscess. World Fournal of Surgery 23, 1191-1194.

Keymer, A. E. and Pagal, M. (1990). Predisposition to helminth infection. In Hookworm Disease : Current Status and New Directions (ed. Schad, G. A. and Warren, K. S.), pp. 177-209. Taylor and Francis, London, UK.

Kirwan, P., Asaolu, S. O., Molloy, S. F., Abiona, T. C., Jackson, A. and Holland, C. V. (2009). Patterns of soil-transmitted helminth infection and impact of four-monthly albendazole treatments in preschool children from semi-urban communities in Nigeria: a double blind placebo-controlled randomised trial. BMC Infectious Diseases 9, 20.

Kuris, A. M., Goddard, J. H. R., Torchin, M. E., Murphy, N., Gurney, R. and Lafferty, K. D. (2007). An experimental evaluation of host specificity: the role of encounter and compatability filters for a rhizocephalan parasite of crabs. International Fournal for Parasitology 37, 539-545.

Lewis, R., Behnke, J. M., Cassidy, J., Stafford, P., Murray, N. and Holland, C. V. (2007). The migration of Ascaris suum larvae, and the associated pulmonary inflammatory response in susceptible C57BL/6j and resistant CBA/Ca mice. Parasitology 134, 1301-1314.

Lewis, R., Stafford, P., Behnke, J. M. and Holland, C. V. (2006). The development of a mouse model to explore resistance and susceptibility to early Ascaris suum infection. Parasitology 132, 289-300.

Loeffler, W. (1932). Zur Differentialdiagnose der Lungen-infiltrierungen. II Ueber fluchtige Succedanininfiltrate (mit Eosinophilie). Beiträge zur Klinik der Tuberkulose 79, 368-382.

Loeffler, W. (1956). Transient lung infiltrations with blood eosinophilia. International Archives of Allergy and Applied Immunity 8, 54-59.

Maizels, R. M. and Kurniawan-Atmadja, A. (2002). Variation and polymorphism in helminth parasites. Parasitology 125, S25-S37.

Maizels, R. M. and Yazdanbakhsh, M. (2003). Immune regulation by helminth parasites: cellular and molecular mechanisms. Nature Reviews Immunology 3, 733-744.

McCallum, H. I. (1990). Covariance of parasite burdens: the effect of predisposition to infection. Parasitology 100, 153-159.

McSharry, C., Xia, Y., Holland, C. V. and Kennedy, M. W. (1999). Natural immunity to Ascaris lumbricoides associated with immunoglobulin E antibody to ABA-1 allergen and inflammation indicators in children. Infection and Immunity 67, 484-489.

Mitchell, G. E., Hogarth-Scott, R. S., Edwards, R. D., Lewers, H. M., Cousins, G. and Moore, T. (1976). Studies on the immune response to parasite antigens mice. 1 Ascaris suum larvae numbers and antiphorylcholine responses in infected mice of various strains and in hypothymic nu/nu mice. International Archives of Allergy and Applied Immunity 52, 64-78.

Montresor, A., Crompton, D. W. T., Gyorkos, T. W. and Savioli, L. (2002). Helminth control in school age children. World Health Organization, Geneva, Switzerland.

Murrell, K. D., Eriksen, L., Nansen, P., Slotved, H.-C. and Rasmussen, T. (1997). Ascaris suum: a revision of its early migratory path and implications for human ascariasis. Fournal of Parasitology 83, 255-260.
Mwangi, T. W., Bethony, J. and Brooker, S. (2007).

Malaria and helminth interactions in humans: an epidemiological viewpoint. Annals of Tropical Medicine and Hygiene 100, 551-570.

Nejsum, P., Roepstorff, A., Anderson, T. J. C., Jorgensen, C. B., Fredholm, M. and Thamsborg, S. M. $(2009 a)$. The dynamics of genetically marked Ascaris suum infections in pigs. Parasitology 136, 193-201.

Nejsum, P., Roepstorff, A., Jorgensen, C. B., Fredholm, M., Goring, H. H. H., Anderson, T. J. C. \& Thamsborg, S. M. (2009b). High heritability for Ascaris and Trichuris infection levels in pigs. Heredity 102, 357-364.

O'Lorcain, P. and Holland, C. V. (2000). The public health significance of Ascaris lumbricoides. Parasitology 121, S51-S71.

Palmer, D. R., Hall, A., Haque, R. and Anwar, K. S. (1995). Antibody isotype responses to antigens of Ascaris lumbricoides in a case-control study of persistently heavily infected Bangladeshi children. Parasitology 111, 385-393.

Peng, W., Zhou, X., Cui, X., Crompton, D. W. T., Whitehead, R. R., Xiong, J., Wu, H., Yang, Y., Wu, W., Xu, K. and Yan, Y. (1998). Transmission and natural regulation of infection with Ascaris lumbricoides in a rural community in China. Fournal of Parasitology 84, 252-258.

Perez, J., Garcia, P. M., Mozos, E., Bautista, M. J. and Carrasco, L. (2001). Immunohistochemical characterization of hepatic lesions associated with migrating larvae of Ascaris suum in pigs. Fournal of Comparative Pathology 124, 200-206.

Petri, W. A., Kirkpatrick, B. D., Haque, R. and Duggal, P. (2008). Genes influencing susceptibility to infection. Fournal of Infectious Diseases 197, 4-6.

Quinnell, R. J. (2003). Genetics of susceptibility to human helminth infection. International Fournal for Parasitology 33, 1219-1231.

Quinnell, R. J., Griffin, J., Nowell, M. A., Raiko, A. and Pritchard, D. I. (2001). Predisposition to hookworm infection in Papua New Guinea. Transactions of the Royal Society of Tropical Medicine and Hygiene 95, 139-142.

Read, A. F. and Viney, M. E. (1996). Helminth immunogenetics: why bother? Parasitology Today 12, 337-343.

Richard, M., Grencis, R. K., Humphreys, N. E., Renauld, J. C. and Van Snick, J. (2000). Anti-IL-9 vaccination prevents worm expulsion and blood eosinophilia in Trichuris muris-infected mice. Proceedings of the National Academy of Sciences, USA 97, 767-772.

Roepstorff, A., Eriksen, L., Slotved, H. C. and Nansen, P. (1997). Experimental Ascaris suum infection in the pig: worm population dynamics following single inoculations with three doses of infective eggs. Parasitology 115, 443-452.

Schad, G. A. and Anderson, R. M. (1985). Predisposition to hookworm infection in humans. Science 228, 1537-1540.

Schopf, L. R., Hoffman, K. F., Cheever, A. W., Urban, J. F. Jr. and Wynn, T. A. (2002). IL-10 is critical for host resistance and survival during gastrointestinal 
helminth infection. Fournal of Immunology 168, 2383-2392.

Shaw, D. J. and Dobson, A. P. (1995). Patterns of macroparasite abundance and aggregation in wildlife populations: a quantitative review. Parasitology 111, S111-S133.

Slotved, H.-C., Eriksen, L., Murrell, K. D. and Nansen, P. (1998). Early Ascaris suum migration in mice as a model for pigs. Fournal of Parasitology 84, 16-18.

Stephenson, L. S. (1987). Ascariasis. In The Impact of Helminth Infections on Human Nutrition (ed. Stephenson, L. S.), pp. 89-127. Taylor and Francis, London and New York.

Tanguay, G. V. and Scott, M. E. (1992). Factors generating aggregation of Heligimosomoides polygyrus (Nematoda) in laboratory mice. Parasitology 104, 519-529.

Tchuente, L. A., Behnke, J. M., Gilbert, F. S., Southgate, V. R. and Vercruysse, J. (2003). Polyparasitism with Schistosoma haematobium and soil-transmitted helminth infections among school children in Loum, Cameroon. Tropical Medicine and International Health 8, 975-986.

Thein-Hlaing, Than-Saw and Myint-Lwin (1987). Reinfection of people with Ascaris lumbricoides following single 6-month and 12-month interval mass chemotherapy in Opko village, rural Burma. Transactions of the Royal Society of Tropical Medicine and Hygiene 81, 140-146.

Thein-Hlaing, Than Saw and Myat Lay Kyin (1991). The impact of three-monthly age-targeted chemotherapy on Ascaris lumbricoides. Transactions of the Royal Society of Tropical Medicine and Hygiene 81, 519-522.

Turner, J. D., Faulkner, H., Kamgno, J., Cormont, F., Van Snick, J., Else, K. J., Grencis, R. K., Behnke, J. M., Boussinesq, M. and Bradley, J. E. (2003). Th2 cytokines are associated with reduced worm burdens in a human intestinal helminth infection. The Fournal of Infectious Diseases 188, 1768-1775.

Urban, J. F. Jr., Katona, I. M., Paul, W. E. and Finkelman, F. D. (1991). Interleukin 4 is important in protective immunity to a gastrointestinal nematode infection in mice. Proceedings of the National Academy of Sciences, USA 88, 5513-5517.

Wakelin, D. and Bradley, J. (2002). Parasite strain diversity and host immune responses. In The Geohelminths: Ascaris, Trichuris and Hookworm (ed. Holland, C. V. and Kennedy, M. W.), pp. 199-218. Kluwer Academic Publishers, The Netherlands.

Wong, M. S., Bundy, D. A. P. and Golden, M. H. N. (1988). Quantitative assessment of geophageous behaviour as a potential source of exposure to geohelminth infection. Transactions of the Royal Society of Tropical Medicine and Hygiene 82, 621-625.

World Health Organization (2002). Report of the WHO informal consultation on the use of Praziquantel during pregnancy and lactation and Albendazole/Medendazole in children under 24 months. WHO, WHO/CPE/PVC 2002.4, Geneva, Switzerland.

Williams-Blangero, S., Subedi, J., Upadhayay, R. P., Manral, D. B., Rai, D. R., Jha, B., Robinson, E. S. and Blangero, J. (1999). Genetic analysis of susceptibility to infection with Ascaris lumbricoides. American Fournal of Tropical Medicine and Hygiene 60, 921-926.

Williams-Blangero, S., VandeBerg, J. L., Subedi, J., Aivaliotis, M. J., Rai, D. R., Upadhayay, R. P., Jha, B. and Blangero, J. (2002). Genes on chromosomes 1 and 13 have significant effects on Ascaris infection. Proceedings of the National Academy of Sciences, USA 99, 5533-5538.

Williams-Blangero, S., VandeBerg, J. L., Subedi, J., Jha, B., Correa-Oiveira, R. and Blangero, J. (2008). Localization of multiple quantitative trait loci influencing susceptibility to infection with Ascaris lumbricoides. The Fournal of Infectious Diseases 197, 66-71.

Yan, M., Marsters, S. A., Grewal, I. S., Wang, H., Ashkenazi, A. and Dixit, V. M. (2000). Identification of a receptor for BlyS demonstrates a crucial role in humoral immunity. Nature Immunology 1, 37-41.

Yazdanbakhsh, M., Kremsner, P. G. and van Ree, R. (2002). Allergy, parasites and the Hygiene Hypothesis. Science 296, 490-494. 